\title{
Radiation-induced anaplastic ependymoma mimicking a skull base meningioma: A case report
}

\author{
ALDO SPALLONE $^{1,2}$, PASQUALE MARCHIONE ${ }^{1}$, MARIO DI CAPUA $^{1}$ and DANIELE BELVISI ${ }^{3}$ \\ ${ }^{1}$ Section of Neurosurgery, Department of Clinical Neurosciences, Neurological Centre of Latium, Rome I-00178; \\ ${ }^{2}$ Department of Biomedicine, University of Rome 'Tor Vergata', Rome I-00173; \\ ${ }^{3}$ Clinical Neurophysiology Unit, Neuromed Institute IRCCS, Pozzilli I-86077, Italy
}

Received March 17, 2015; Accepted October 29, 2015

DOI: $10.3892 / \mathrm{etm} .2015 .2945$

\begin{abstract}
The present study describes the case of a 63-year-old woman presenting with headache, dizziness and vomiting due to a an ovoid mass in the left pre-bulbar cistern, apparently arising from the lower clivus and the foramen magnum. The clinical history revealed the subtotal removal of a right cerebellar low-grade glioma 15 years previously and subsequent conventional 60-Gy radiotherapy. Notably, following gross total resection, histopathological examination showed microscopic features that resulted in a diagnosis of anaplastic ependymoma. The patient underwent surgery to remove the mass and post-operative chemotherapy with temozolomide. A progressive improvement of neurological signs and symptoms was observed during the postoperative course. At the 6-month follow-up, the patient was free from clinical and radiological recurrence. The unusual features of this rare secondary brain tumor were the extrassial location in the posterior fossa, the unusual age-associated location of the histological subtype and the fact that it closely mimicked a skull-base meningioma.
\end{abstract}

\section{Introduction}

Although radiotherapy remains a key treatment of central nervous system (CNS) tumors in both children and adults, secondary malignancies have been documented as potential rare long-term adverse effects of radiation, the risk of which has not been reduced by newer protocols and stereotactic radiosurgery (1). According to diagnostic criteria extrapolated from Cahan's definition of radiotherapy-related systemic neoplasm, a diagnosis of secondary brain malignancies is possible when: i) tumors are located in a prior radiation field, ii) an adequate latency period, usually years, passes between the radiation therapy and the onset of the secondary tumor, iii) the new

Correspondence to: Dr Daniele Belvisi, Clinical Neurophysiology Unit, Neuromed Institute IRCCS, 18 Via Atinense, Pozzilli I-86077, Italy

E-mail: dbelvisi@hotmail.it

Key words: post irradiation tumors, skull base approach, radiation-induced meningioma, radiation-induced ependymoma neoplasm differs histopathologically from the primary disease, and iv) any carcinogenic disease such as tuberous sclerosis and neurofibromatosis has been excluded (2). Since the first reported cases of radiation-induced CNS tumors in the early 1950s, meningiomas have been the most frequently observed tumor type, but low-or high-grade gliomas and sarcomas have been also been described (1,3). Anaplastic ependymoma is a rare subtype of ependymoma, particularly in adults, that frequently has an extraventricular and ectopic supratentorial location $(4,5)$. By contrast, intra- and extraventricular ependymomas of the posterior fossa most often arise in children $(5,6)$. A total of 76 cases of ectopic ependymoma have previously been reported, of which 53 were intraspinal tumors and 23 were intracranial supratentorial tumors; 9/23 cases of ectopic supratentorial ependymoma exhibited anaplastic characteristics (4-6). To the best of our knowledge, only one case of post-irradiation anaplastic ependymoma has previously been described in the literature, with a remarkable clinical response to temozolomide (7). The present study reports an unusual case of a radiation-induced ectopic anaplastic subependymoma mimicking a skull base meningioma.

\section{Case report}

A 63-year-old woman was admitted from the Emergency Room to the Department of Clinical Neurosciences at the Neurological Centre of Latium (Rome, Italy) with a drug-resistant fronto-temporal headache and dizziness associated with incoercible vomiting without nausea. Neurological examination showed static and dynamic axial ataxia associated with dysphonia. The clinical history of the patient reported a previous neurosurgical intervention for the subtotal removal of a right cerebellar low-grade glioma 15 years earlier at a different institution. A ventricular-peritoneal shunt was positioned at the same time because of hydrocephalus. Subsequently, the patient had been treated with conventional 60-Gy radiotherapy.

Acutely performed no-contrast computed tomography scanning of the brain revealed a mass-occupying solid lesion in left side of the lower clivus. The patient rapidly underwent contrast-enhanced magnetic resonance imaging (MRI) of the brain, which showed an ovoid T2-hyperintense and T1-hypointense mass of dimensions $25 \times 20 \times 13 \mathrm{~mm}$ in the 
A

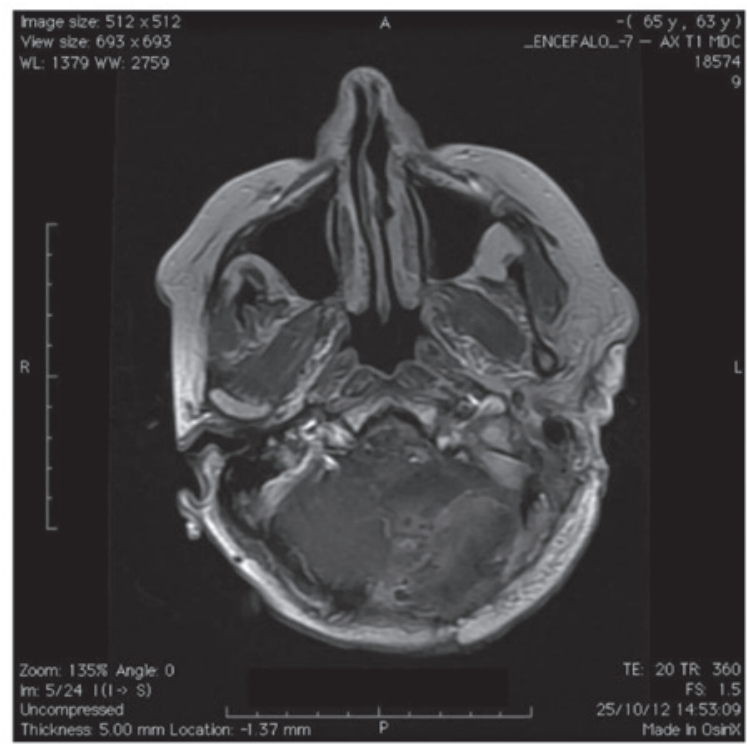

B

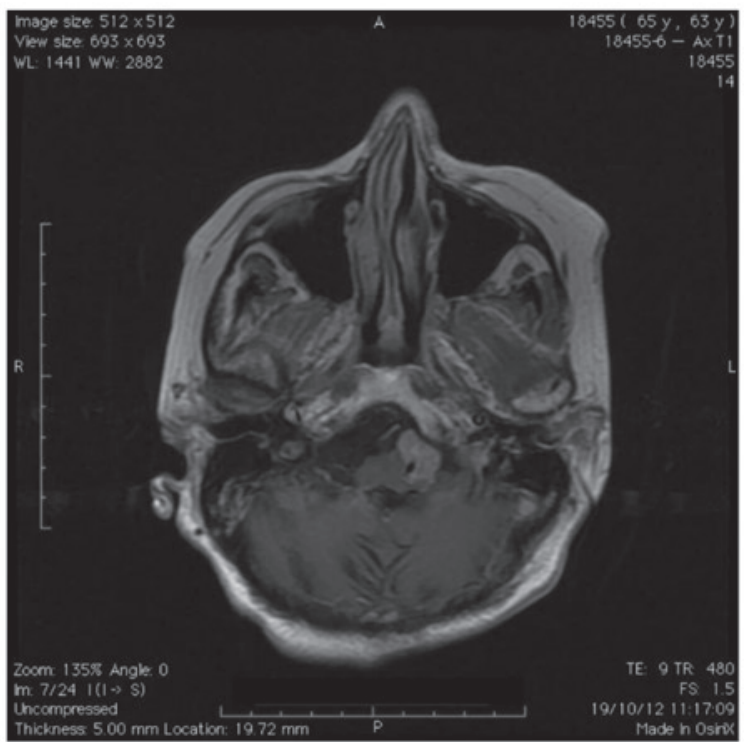

Figure 1. (A) Pre-operative magnetic resonance image (MRI) shows an ovoid-shaped highly enhanced mass in the left side of the foramen magnum. (B) Post-operative MRI shows the apparent total removal of the mass.

left pre-bulbar cistern, apparently arising from the left side of the lower clivus and the foramen magnum (Fig. 1A). The lesion reached the left anterior-lateral portion of the lower brainstem, which was shifted controlaterally and showed a homogeneous enhancement after contrast injection. The left vertebral artery was entrapped in the mass, however without any narrowing of the lumen. In addition, the ventricular shunt appeared correctly placed in the frontal horn of the right lateral ventricle. Radiological features and the clinical history of the patient suggested a post-irradiation meningioma of the lower clivus. The patient underwent microsurgical resection via a far lateral approach to the foramen magnum by means of a limited condylar resection and displacement of the extradural vertebral artery that allowed an excellent exposure of the tumor. Intraoperatively, the macroscopic aspect of the lesion confirmed the suspicion of a meningioma; however, a dural attachment was not found in spite of the neuroradiological appearance. The dissection was challenging because of the close relationship to the lower cranial nerves and the firm adhesion to the vertebral artery. However, an almost gross total resection was possible, with the exception of only a very small fragment, closely adherent to the arterial wall. Nevertheless, post-operative contrast-enhanced MRI of the brain showed an apparently complete removal of the lesion (Fig. 1B). Notably, histopathological examination showed a high cellularity with typical perivascular pseudorosettes. Immunochemical analysis revealed increased nuclear atypia and vascular proliferation, and a Ki67 level of $>20 \%$. These microscopic features resulted in a diagnosis of anaplastic ependymoma (Fig. 2).

The postoperative course of the patient was characterised by a progressive improvement of ataxia without any other neurological signs and symptoms. Since radiation therapy was not recommended, the patient underwent chemotherapy with temozolomide. At the 6-month follow-up, the patient was free from clinical and radiological recurrence. Written informed consent was obtained from the patient for publication of this case report and the accompanying images.

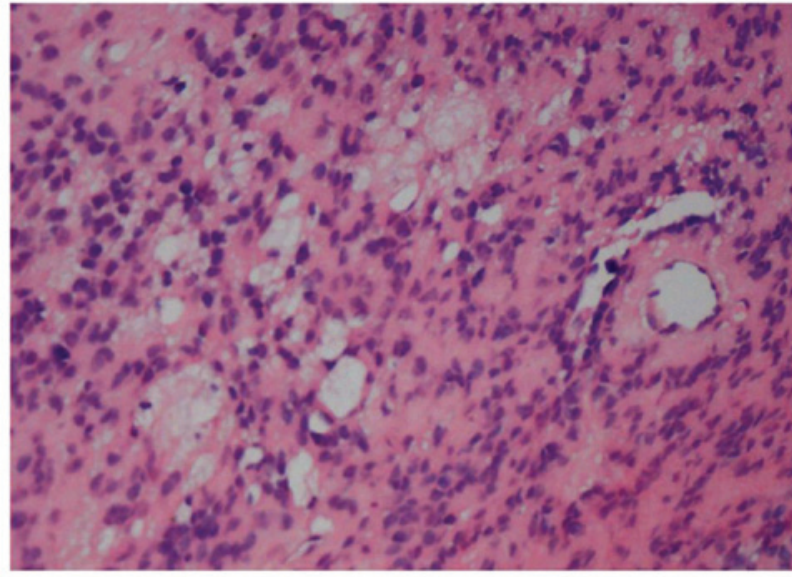

Figure 2. Photomicrograph showing an anaplastic ependymoma, with high cellularity and typical perivascular pseudorosettes. Mitotic activity is evident (magnification, x100).

\section{Discussion}

Although rare, a secondary brain tumor has to be considered in a long-term follow up of patients treated with radiotherapy $(1,8)$. In the present case, the observed lesion fulfilled the extrapolated Cahan's criteria for a radiation-induced brain tumor: Site location in the previously irradiated area; a sufficiently long time interval; a hystologically different nature from the primary one; and the absence of pathologies favoring the development of tumors such as von Recklinghausen's disease (2). The main features that make the present case unusual are the morphological characteristics of the lesion at neuroimaging and the unusual location of the histological subtype for a patient of this age. In particular, the extra-assial location of the tumor in the left pre-bulbar cistern, apparently arising from the left side of the lower clivus and the foramen magnum, suggested more intuitively a diagnosis of post-radiation meningioma. According to the literature, secondary meningiomas have a 
strong tendency to an aggressive biological behavior and a higher recurrence rate than spontaneous meningiomas, with a slight male predominance $(1,3,9,10)$. Such an association with occlusion of the larger intracranial arteries has been reported as a result of extracranial radiation. By contrast, gliomas following irradiation, as post-traumatic gliomas $(11,12)$ have been more rarely described and have a higher incidence in younger patients (60-75\% of cases) (1). In a previous study it was demonstrated that, following radiotherapy, gliomas developed within both the full-dose prescription volume and in the lower-dose periphery, suggesting a non linear relationship between radiation dose and risk of secondary glioma and a more relevant role of dose volume in risk definition (8). Radiation therapy for brain tumors and leukemia in childhood has been significantly associated with the occurrence of gliomas with a markedly longer latency period, as compared with meningiomas (6). Furthermore, radiation-induced high-grade gliomas and secondary glioblastomas more often occur in the supratentorial location and the cerebellum than primitive ones (13). To our knowledge, only a few cases of post-radiation ependymoma have been reported. Among them only one case was an anaplastic subtype (7). This occurred in a middle-aged patient with a right caudate anaplastic ependymoma associated with a right temporal meningioma and a left frontal cavernous malformation following radiation treatment for a pituitary macroadenoma (14). All other cases are relatively young with a diagnosis of the first neoplasm in childhood or in adolescence. A radiation-induced anaplastic ependymoma has also been described in a 25-year-old woman with a marked clinical response to temozolomide (7). Ependymomas are relatively uncommon tumors of the CNS with a prevalence of $1.2-7.8 \%$ of all intracranial neoplasms and $2-6 \%$ of all gliomas (5). They represent the most frequent primary brain tumors after pilocytic astrocytomas and medulloblastomas in children, but are particularly rare in adults $(5,6)$. Usually they arise from the cells lining the ventricular system and central canal in the spinal cord, whilst the ectopic variant starting from brain parenchyma has been sporadically observed in various locations $(4,8)$. The malignant form, namely anaplastic ependymoma, is a high-grade glioma with ependymal differentiated cells and high mitotic activity. A typical microvascular proliferation and necrosis with perivascular pseudorosettes has frequently been reported $(13,14)$.

In the present case, the high cellularity, perivascular pseudorosettes and high mitotic index confirmed a histological diagnosis of anaplastic ependymoma. In contrast with previous reports, the present patient was not young and the lesion was atypically located in the posterior fossa. Supratentorial and infratentorial anaplastic ependymomas exhibit different histopathological behaviours; the former are more frequently high-grade tumors (50-60\% of cases) than the latter (20-40\% of cases) and show a typical age-related distribution $(1,4,5)$. Supratentorial and intra-medullary ependymomas are more frequent in adults whilst the posterior fossa location is typical of childhood (4-6). Moreover, an ectopic location is more frequent in supratentorial than in infratentorial ependymomas (5).

Although the risk/benefit ratio is more than acceptable, the likelihood of developing a secondary tumor in an intracranial regions previously irradiated for therapeutic purposes requires consideration, although it is relatively rare in comparison with other long-term complications of radiotherapy, such as recurrence of the primary lesion and necrosis of the irradiated tissue. Earlier reports have suggested that low-dose ionising irradiation increases the risk of meningiomas, while radiation-induced gliomas are uncommon. The present case is exceptional for several reasons. First, the lesion closely mimicked a skull base meningioma; secondly, it required the same difficult intraoperative dissection as would have a real meningioma, but was later identified to be an ependymoma; and finally, it occurred in a location and in an age range quite different from the usual ones for similar histological subtypes. For irradiation-induced malignant tumors, the therapeutic options following neurosurgery are limited to chemotherapy due to the prior exposure to radiotherapy. The results of treatment in the present case were very good, although the follow-up is very short and recurrence is possible in the future.

\section{References}

1. Ecemis GC, Atmaca A and Meydan D: Radiation-associated secondary brain tumors after conventional radiotherapy and radiosurgery. Expert Rev Neurother 13: 557-565, 2013.

2. Cahan WG and Woodard HQ: Sarcoma arising in irradiated bone; report of 11 cases. Cancer 1: 3-29, 1948.

3. Waga $\mathrm{S}$ and Handa H: Radiation-induced meningioma: With review of literature. Surg Neurol 5: 215-229, 1976.

4. Schwartz TH, Kim S, Glick RS, Bagiella E, Balmaceda C, Fetell MR, Stein BM, Sisti MB and Bruce JN: Supratentorial ependymomas in adult patients. Neurosurgery 44: 721-731, 1999.

5. Mork SJ and Loken AC: A follow-up study of 101 cases. Cancer 40: 907-915, 1977

6. Coulon RA and Till K: Intracranial ependymomas in children: A review of 43 cases. Childs Brain 3: 154-164, 1977.

7. Khoo HM, Kishima H, Kinoshita M, Goto Y, Kagawa N Hashimoto N, Maruno M and Yoshimine T: Radiation-induced anaplastic ependymoma with a remarkable clinical response to temozolomide: A case report. Br J Neurosurg 27: 259-261, 2013.

8. Salvati M, Frati A, Russo N, Caroli E, Polli FM, Minniti G and Delfini R: Radiation-induced gliomas: Report of 10 cases and review of the literature. Surg Neurol 60: 60-67, 2003.

9. Spallone A, Gagliardi FM and Vagnozzi R: Intracranil meningiomas related to external cranial radiation. Surg Neurol 12: 153-159, 1979.

10. Umansky F, Shoshan Y, Rosenthal G, Fraifeld S and Spektor S: Radiation-induced meningioma. Neurosurg Focus 24: E7, 2008.

11. Spallone A, Izzo C and Orlandi A: Post traumatic glioma: Report of a case. Case Rep Oncol 7: 403-409, 2013.

12. Spallone A, Neroni M and Giuffrè R: Multiple skull base meningioma: Case report. Surg Neurol 51: 274-280, 1999.

13. Paulino AC, Mai WY, Chintagumpala M, Taher A and Teh BS: Radiation-induced malignant gliomas: Is there a role for reirradiation? Int J Radiat Oncol Biol Phys 71: 1381-1387, 2008.

14. Alexander MJ, DeSalles AA and Tomiyasu U: Multiple radiation-induced intracranial lesions after treatment for pituitary adenoma. Case report. J Neurosurg 88: 111-115, 1998. 